\title{
THIRD-BODY PERTURBED APSIDAL MOTION IN ECLIPSING BINARIES
}

\author{
T. Borkovits ${ }^{1}$, E. Forgács-Dajka ${ }^{2}$ and S.A. Rappaport ${ }^{3}$
}

\begin{abstract}
Tidally forced apsidal motion in eccentric eclipsing binaries is a key observable of stellar structure studies, and may serve as important probe for different tidal dissipation theories. There are, however, alternative physical processes which result in additional contributions to the observed apsidal motion. Here we mainly concentrate on the perturbing effect of a third, distant stellar companion, forming a hierarchical triple system with the eclipsing pair. First we discuss the mathematical form of the combined third body and tidally forced apsidal motions, and its observational aspects. Then we present a brief description of an eclipse timing variation study (based on the above mentioned analytical formulae) of 26 Kepler spacecraft-discovered 3-rd body perturbation-dominated compact hierarchical triples.
\end{abstract}

\section{Introduction}

The apsidal motion effect (AME) is a well-observable phenomenon in eccentric eclipsing binaries (eEB) since it gives rise to characteristic and, in many cases, large amplitude changes in the apparent eclipsing period and, therefore, real eclipse timing variations (ETV). This effect may have at least three different origins as (i) the non-spherical mass distribution of the tidally distorted binary components; (ii) the general relativistic apsidal motion; and (iii) the gravitational perturbations of a (not too distant) ternary stellar component, which may be present in a significant portion of binary stars (see, e.g., Tokovinin et al. 2006). The significance of observations of the AME in the context of tidal interactions in close binaries was reviewed by Mazeh (2008).

\footnotetext{
${ }^{1}$ Baja Astronomical Observatory of Szeged University, H-6500 Baja, Szegedi út, Kt. 766, Hungary

${ }^{2}$ Department of Astronomy, Eötvös University, H-1118 Pázmány Péter stny. 1/A, Budapest, Hungary

${ }^{3}$ M.I.T. Department of Physics and Kavli Institute for Astrophysics and Space Research, 70 Vassar St.,Cambridge, MA 02139, USA
} 
Apsidal motion (or, more generally, any long-term third-body perturbations) in a hierarchical triple system has been intensively studied in the past two decades when it became clear that triplicity may play a significant role in the evolution of binary systems from the earliest moments of their formation (see, e.g., Fabrycky \& Tremaine 2007) to the very end of stellar evolutions (see e.g. Tauris $\&$ van den Heuvel 2014). Most of these papers, however, focussed on the consequences of the third-body perturbations on the evolutionary important longer (thermal or, even nuclear) timescales. (A recent review of the field is given by Naoz 2016.)

In the last decade, however, the advent of ultra-precise space photometry and especially, the operation of the Kepler space telescope (see, e.g., Borucki et al. 2010) has revolutionized the field of EB studies and amongst other unexpected results, has led to the discovery of dozens of eEBs with relatively close and, therefore, strongly interacting third components.

In this paper we concentrate on the observational apsects of the short-term effects of a third, perturbing companion star on the observable apsidal advance rates of an eccentric eclipsing binary.

\section{Mathematical description of the apsidal motion forced eclipse timing variations}

In the case of an eEB the orientation of the orbit with respect to the observer strongly affects the orbital phase and, therefore, the time when eclipses occur. Apart from a very weak inclinations dependence (which can be eliminated in all the recently known eEBs), the apsidal motion cotribution to the ETV can be given in the following, exact, analytical form (see, e.g., Giménez \& Garcia-Pelayo 1983):

$$
\Delta_{\text {apse }}= \pm \frac{P_{1}}{\pi}\left[\arctan \left(\frac{e_{1} \cos \omega_{1}}{1+\sqrt{1-e_{1}^{2}} \mp e_{1} \sin \omega_{1}}\right)+\sqrt{1-e_{1}^{2}} \frac{e_{1} \cos \omega_{1}}{1 \mp e_{1} \sin \omega_{1}}\right],
$$

where $P_{1}$ denotes the anomalistic period of the $\mathrm{EB}, e_{1}$ stands for its eccentricity, while $\omega_{1}$ is the argument of periastron of the relative orbit of the secondary component with respect to the primary, expressed in the so-called observational frame of reference, the fundamental plane of which is the plane of the sky (i.e. the plane parallel to the line of sight of the binary), and $\omega_{1}$ is measured from the intersection of this plane with the orbital plane of the EB. Finally, the upper and lower signs refer to the primary and secondary eclipses, respectively.

If an eEB was subjected to apsidal motion, the observable argument of periastron $\omega_{1}$ would vary in time, and the occurrence times of the primary and secondary eclipses would exhibit almost anticorrelated ETVs. The time dependence of the ETVs determined by the function $\omega_{1}(t)$, the characteristics of which depends on the physical origin of the apsidal motion. 


\subsection{The two classical types of AME}

Both relativistic and classical tidal (non-dissipative) AMEs result in a prograde ${ }^{1}$ apsidal revolution with a constant speed. Furthermore, as far as we omit the effects of tidal dissipation, which are only effective on much longer timescales (see, e.g., Zahn 2008, for a review), neither the anomalistic period, nor the eccentricity are affected in these cases. Therefore, for most of the eEBs known in the pre-Kepler era one can write that

$$
\omega_{1}(E)=\omega_{1}(0)+\Delta \omega_{1} E
$$

where $\Delta \omega_{1}$ means the constant variation in $\omega_{1}$ during one orbital period, and $E$ is the cycle number.

As far as only these two kinds of apsidal motions are effective in an eEB, the apsidal advance rate can, in principle, be determined (even in the absence of one fully covered period) from Equation (2.1). The problem, however, is that usually these two kinds of AME produces very long apsidal motion periods. For relativistic AME the nominal period can be converted into

$$
U_{\mathrm{GR}}[\mathrm{yr}] \approx 1800 \times\left(1-e_{1}^{2}\right)\left(\frac{m_{\mathrm{AB}}}{m_{\odot}}\right)^{-2 / 3} P_{1}^{5 / 3}[\mathrm{~d}]
$$

(see, e.g., Weinberg 1972), while the tidal component for two similar, 'pseudosynchronously' rotating stars leads to the nominal period of

$$
U_{\text {tide }}[\mathrm{yr}] \approx 10.65 \times \frac{\left(1-e_{1}^{2}\right)^{5}}{1+\frac{81}{34} e_{1}^{2}+\frac{75}{36} e_{1}^{4}-\frac{55}{17} e_{1}^{6}}\left(\frac{m_{\mathrm{AB}}}{m_{\odot}}\right)^{5 / 3}\left(k_{2}^{*}\right)^{-1}\left(\frac{R}{R_{\odot}}\right)^{-5} P_{1}^{13 / 3}[\mathrm{~d}],
$$

where $k_{2}^{*}=100 k_{2}$ was used for a better representation of the magnitude of the constant, as for main-sequence stars $0.1 \leq k_{2}^{*} \leq 3$. (For getting this equation the functional form of the apsidal advance rate was taken from Cowling 1938 and Sterne 1939, and the pseudo-synchronous rotation rate was obtained from Hut 1981.) Thus, considering even a relatively close $\left(P_{1}=5 \mathrm{~d}\right)$ pair formed by two early-type stars $\left(m_{\mathrm{A}}=m_{\mathrm{B}}=3 m_{\odot}\right)$, revolving around each other on a significantly eccentric orbit $\left(e_{1}=0.5\right)$, these periods become $U_{\mathrm{GR}} \approx 6000 \mathrm{yr}$ and $U_{\text {tide }} \approx$ $1200 \mathrm{yr}$, resulting in a net period of AME $U \approx 1000$ yr. Furthermore, as one can see, the significance of the tidal effect decreases very quickly towards larger orbital separations and, finally, for wider eEBs with periods $\left(P_{1} \gtrsim 10 \mathrm{~d}\right)$, AME will be predominated by the relativistic effect.

Therefore, for most of the eEBs and especially for those having a significant relativistic contribution, observations are available only for a very small fraction of the full period. In this case, instead of (2.1) it is better to use the difference of the

\footnotetext{
${ }^{1}$ For highly inclined rotational axes, however, it may happen that the tidal contribution becomes negative, i.e. gives a retrograde contribution, as it was likely found in the case of DI Her (Albrecht et al. 2009). In this paper we neglect this rare possibility.
} 
two ETV curves, i.e. the time-offset of the secondary eclipses from the mid-times of the previous and the forthcoming primary eclipses:

$$
D=-\frac{P_{1}}{\pi}\left[2 e_{1} \cos \omega_{1}-\left(\frac{1}{3} e_{1}^{3}+\frac{1}{8} e_{1}^{5}\right) \cos 3 \omega_{1}+\frac{3}{40} e_{1}^{5} \cos 5 \omega_{1}\right]+\mathcal{O}\left(e_{1}^{7}\right)
$$

As one can see, as far as $e_{1}$ and/or the actual value of $\omega_{1}$ is known e.g. from radial velocity and/or photometric lightcurve solutions, the slope of the measured section of function $D$ can be converted into the apsidal advance rate, as

$$
\dot{D} \approx \frac{P_{1}}{\pi} 2 e_{1} \dot{\omega}_{1} \sin \omega_{1}+\mathcal{O}\left(e_{1}^{3}\right)
$$

In this way, after the subtraction of the theoretically expected relativistic contribution, finally one can infer the apsidal motion constants $k_{2}$ (related to the inner stellar structure). Conversely, if the latter is known, the results can be used for probing the GR theory. Both directions were applied historically for several cases.

\subsection{Third body induced AME}

For third component induced dynamical apsidal motion this simple picture changes radically. In this case, in general, none of the orbital elements remain constant (except for the semimajor axes). This is especially true for systems with high mutual inclination and negligible tidal oblateness, where, e.g. the eccentricity of the inner binary might be subject to very high amplitude cyclic variations, and the apsidal line may rotate, or alternatively, librate also with strongly varying speed. (This is the so-called Kozai-Lidov mechanism, see Naoz 2016 for a recent review.) It was found, however, already by Söderhjelm (1984) that tidal interactions between the binary members can significantly alter the dynamics of such triple systems.

In a hierarchical triple system periodic third-body perturbations can be divided into three groups according to their characteristic timescales and relative amplitudes (Brown 1936). The shortest period perturbations have a timescale of $P_{1}$ and relative amplitude of $\left(P_{1} / P_{2}\right)^{2}$, the medium category has timescale of $P_{2}$ and amplitude of $P_{1} / P_{2}$, while the so-called apse-node perturbations have a characteristic timescale of $P_{2}^{2} / P_{1}$ with a relative amplitude of unity.

First we consider the last group. The leading term of the third-body forced observable apsidal advance rate takes the form ${ }^{2}$ (see, e.g., Borkovits et al. 2007):

$$
\begin{aligned}
\left(\frac{\mathrm{d} \omega_{1}}{\mathrm{~d} t}\right)_{3 \mathrm{~b}} & =\frac{3 \pi}{4} \frac{m_{\mathrm{C}}}{m_{\mathrm{ABC}}} \frac{P_{1}}{P_{2}^{2}} \frac{\left(1-e_{1}^{2}\right)^{1 / 2}}{\left(1-e_{2}^{2}\right)^{3 / 2}}\left(3 \cos ^{2} i_{\mathrm{m}}-1+5 \sin ^{2} i_{\mathrm{m}} \cos 2 g_{1}\right) \\
& =A_{3 \mathrm{~b}}+B_{3 \mathrm{~b}} \cos 2 g_{1},
\end{aligned}
$$

where $i_{\mathrm{m}}$ stands for the mutual (or relative) inclination of the planes of the inner and outer orbits. Furthermore, $g_{1}$ denotes the argument of periastron of the eEB

\footnotetext{
${ }^{2}$ Note that we omitted the indirect term occuring from the orbital plane precession in noncoplanar systems, as it has a practically negligible contribution in eclipsing systems.
} 
in the so-called dynamical frame of reference. The fundamental plane of this system is the invariable plane of the triple system, and $g_{1}$ is measured from the intersection of the orbital and the invariable planes. The two kinds of argument of periastron have the relation

$$
\omega_{1}=g_{1}+n_{1},
$$

where the longitude-like quantity $n_{1}$ measures the arc distance between the ascending nodes of the eEB's orbital planes in the two different systems of references. For $g_{1}$ (which, hereafter will be referred as the dynamical argument of periastron) the perturbation equation gets an analogous form as

$$
\left(\frac{\mathrm{d} g_{1}}{\mathrm{~d} t}\right)=A_{\mathrm{GR}}+A_{\mathrm{tide}}+A_{\mathrm{g} 1}+B_{\mathrm{g} 1} \cos 2 g_{1}
$$

where the relativistic and tidal terms are the same as for $\dot{\omega}_{1}$, while $A_{\mathrm{g} 1}$ and $B_{\mathrm{g} 1}$ differ slightly from their counterparts in the observational system. The perturbed dynamical apsidal advance rate can be obtained from the (usually complicated) solution of this equation. For the discussion of prompt observational effects, we are interested in the instantaneous third-body apsidal advance rate, instead of the complete $g_{1}(t)$ or $\omega_{1}(t)$ solution. More important, however, in our point of view, is that, as mentioned before, $e_{1}$ will also vary in time in case of third star perturbations, according to the relation:

$$
\frac{1}{e_{1}}\left(\frac{\mathrm{d} e_{1}}{\mathrm{~d} t}\right)=B_{3 \mathrm{~b}} \sin 2 g_{1} .
$$

First we consider the scenario where the tidal and the third-body perturbations are of the same order of magnitude, and having a net period much longer than the length of the observations. In this case the combination of Equations (2.7) and (2.10) gives for the instantaneous slope of the differential ETV curve $D$ :

$$
\begin{aligned}
\dot{D} & \approx \frac{P_{1}}{\pi} 2 e_{1}\left[A \sin \omega_{1}-B \sin \left(\omega_{1}-2 n_{1}\right)\right] \\
& \approx \frac{P_{1}}{\pi} 2 e_{1} \sqrt{A^{2}+B^{2}-2 A B \cos 2 n_{1}} \sin \left(\omega_{1}+\phi\right),
\end{aligned}
$$

where we used the simplified denotations $A \equiv A_{\mathrm{GR}}+A_{\mathrm{tide}}+A_{3 \mathrm{~b}}$ and, $B \equiv B_{3 \mathrm{~b}}$ and furthermore,

$$
\tan \phi=\frac{B \sin 2 n_{1}}{A-B \cos 2 n_{1}} .
$$

In this short note, instead of a full discussion we consider only some interesting cases. Taking first an (almost) coplanar situation (i.e. $i_{\mathrm{m}} \approx 0^{\circ}$ ) one finds that $B \approx 0$ and $A_{3 \mathrm{~b}}>0$. Thus, in this case there are no significant eccentricity variations and (2.11) reduces again to (2.6). The inferred instantaneous apsidal advance rate, however, may be significantly larger (and consequently, the apsidal motion period shorter) than in the absense of the third component. 
Considering the other end, the scenario of near-perpendicular orbits (with $i_{\mathrm{m}} \approx$ $90^{\circ}$ ), here we discuss the two extrema, namely (i) when both the inner and outer orbits are perpendicular to the plane of the sky, and (ii) when the outer orbit fits more or less to the plane of the sky.

In the first case $n_{1} \approx \pm 90^{\circ}$, while in the second case either $n_{1} \approx 0^{\circ}$ or $n_{1} \approx$ $180^{\circ}$. Both configurations lead to $\phi=0^{\circ}$, therefore the trigonometric part of (2.11) reduces again to $\sin \omega_{1}$. The amplitude, however, would no longer give directly $\dot{\omega}_{1}$, because $\dot{e}_{1} \neq 0$. However, as far as the exictence of a third, perturber companion remained unknown, this amplitude, inferred from the observations, would be falsely identified with the apsidal advance rate. In such a case the two extrema would lead to the following false apsidal advance rates:

$$
\left(\dot{\omega}_{1}\right)_{\mathrm{false}} \approx A_{\mathrm{GR}}+A_{\mathrm{tide}} \pm \frac{9 \pi}{2} \frac{m_{\mathrm{C}}}{m_{\mathrm{ABC}}} \frac{P_{1}}{P_{2}^{2}} \frac{\left(1-e_{1}^{2}\right)^{1 / 2}}{\left(1-e_{2}^{2}\right)^{3 / 2}} .
$$

As one can see, the first situation would result a faster than expected false apsidal advance rate, while the second configuration would give a too slow apsidal advance rate. From an observational point of view, however, there are at least two important differences between the two configurations. In the first case, where both orbits are perpendicular to the sky (i.e. both observable inclinations are about $90^{\circ}$ ), the revolution of the eEB around the common centre of mass of the triple system would result in both cyclic light-travel time effect in the ETVs and variations in the systemic radial velocity, which would reveal the presence of the third component. Oppositely, in the second situation the revolution of the eEB around the common centre of mass would happen in the plane of the sky, and therefore, these effects would not occur. Furthermore, it can also be shown that the variation of the observable inclination which is a direct consequence of the precession of the orbital planes in non-coplanar triples has a maximum speed about $n_{1} \approx \pm 90^{\circ}$ (i.e. in the first case), and takes its minimum about $n_{1} \approx 0^{\circ}$ and $\approx 180^{\circ}$ (see, e.g., Borkovits et al. 2015). This again emphasises that in such a configuration a third perturber might go unnoticed with a significantly increased probability.

It is possible to give a crude estimation of the periods $P_{2}$ of those unnoticed third companions that could lead to false apsidal advance rates. For simplicity we omit the relativistic contribution. From the condition that only a small part of one complete apsidal revolution was covered one can suppose that, let's say, $U_{\text {tide }} \geq 10^{3}$ yr. Furthermore, we expect the ratio $\left(\mu=U_{3 \mathrm{~b}} / U_{\text {tide }}\right)$ to be of the order of unity. Then, substituting $1 / 3$ into the mass ratio, and neglecting the eccentricity dependences of (2.13) one can estimate that

$$
P_{2} \lesssim 500 \times \sqrt{\mu} \sqrt{P_{1}} .
$$

Consequently, for a $P_{1} \simeq 5$ day-period eEB (where $U_{\text {tide }}$ is expected to exceed 1000 years), the effect of a third companion revolving on a $P_{2} \lesssim 1000$-day orbit 

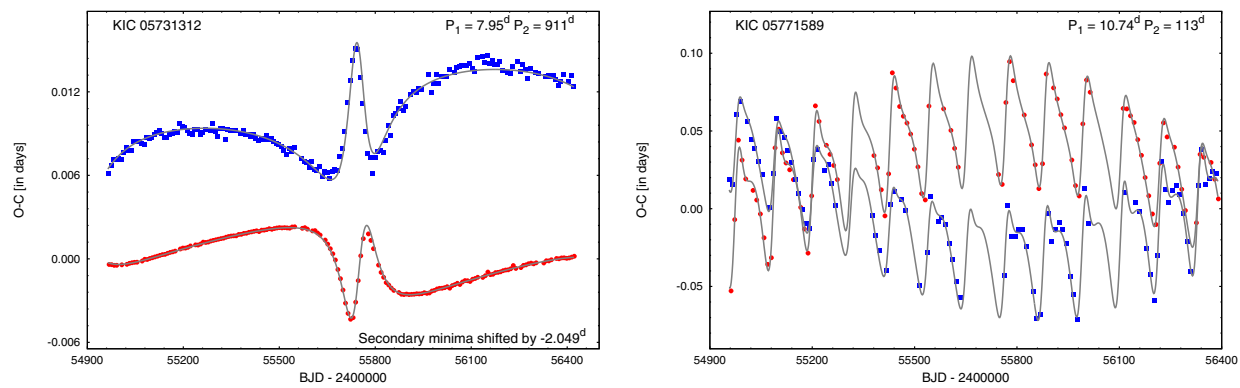

Fig. 1. Typical third-body dynamical interactions dominated ETV curves of two eEBs in the primordial Kepler field. Red circles and blue boxes denote primary and secondary eclipses, respectively. The best-fitted analytic solutions of Borkovits et al. (2015) are drawn with grey lines.

may exceed the tidal contribution. For larger inner periods this limit (for the same triple) grows with

$$
P_{2}^{\mathrm{equ}} \propto P_{1}^{8 / 3}
$$

i.e., the third-body effect becomes quickly predominant even for more distant third components.

\section{Eccentric EBs with dominant third-body perturbations in the prime Kepler field}

As was shown above, if an eEB had a close stellar companion with a period less than 1-2 years, then the strength of the third-body forces would strongly exceed both those of the tidal and relativistic interactions. No such kinds of triples were known before the Kepler-mission. Recently, however, our ETV analysis of 26 eEBs with third stellar companion candidates in the prime Kepler-field (Borkovits et al. 2015) found that in all of these systems the third-body contributions are much larger than the tidal and relativistic components. Furthermore, in these triples the short-term (relative to the AME period) behaviour of the ETVs is dominated by the $P_{2}$-timescale perturbations instead of the 'apse-node'-timescale ones. These effects produce very complex ETV-s, the symbolic form of which is

$$
\Delta_{\mathrm{dyn}}=\frac{1}{2 \pi} \frac{m_{\mathrm{C}}}{m_{\mathrm{ABC}}} \frac{P_{1}^{2}}{P_{2}}\left(1-e_{2}^{2}\right)^{-3 / 2} f\left(e_{1}, \omega_{1}, i_{\mathrm{m}}\right) \mathcal{S}\left(v_{2}, \omega_{2}, g_{1}, g_{2}\right),
$$

where $v_{2}$ in the argument of the trigonometric terms $(\mathcal{S})$ refers to the true anomaly of the third star (for the full equations see Borkovits et al. 2015). The combination of the AME (2.1), dynamical (3.1) and the classical light-travel time (Irwin 1952) ETV contributions allowed us not only to give qualitative interpretations of the never-before-seen-shaped ETV curves (Fig. 1), but also made it possible to determine the complete spatial configurations of these triples. Furthermore we were 
also able to determine the dynamical masses of the stellar components, though with greater uncertainties. For five eEBs with medium relative inclinations we inferred retrograde apsidal motion. These eEBs should also be subject to large amplitude eccentricity variations, and may be promising targets for future tidal third-body interaction studies.

\section{Conclusion}

We have discussed the observational consequences of dynamical apsidal motion (induced by the gravitational perturbations of a third companion star) in an eEB. We have shown that an unnoticed third star with an orbital period of a few years may result in badly interpreted apsidal motion observations with systematic biases towards calculating much longer apsidal motion periods than what should be theoretically expected.

For shorter outer period sytems with predominant third-body perturbations, the ETVs of such eEBs carry significant extra information that can be used to determine the full 3D orbital configurations, as well as the masses of these systems. This latter approach was susseccfully applied to some dozens of eEBs in the primoridal field of the Kepler spacecraft.

T.B. and E.F.-D. thanks for the organizers their kindest invitation and hospitality. This work has been partially supported by the Hungarian NKFIH-OTKA Grant K-113117.

\section{References}

Albrecht, S., Reffert, S., Snellen, I.A.G., \& Winn, J.N., 2009, Nature, 461, 373

Borkovits, T., Forgács-Dajka, E., \& Regály, Zs., 2007, A\&A, 473, 191

Borkovits, T., Rappaport, S., Hajdu, T., \& Sztakovics, J., 2015, MNRAS, 448, 946

Borucki, W.J., Koch, D., \& Basri, G., et al., Science, 327, 977

Brown, E.W., 1936, MNRAS, 97, 62

Fabrycky, D., \& Tremaine, S., 2007, ApJ, 669, 1298

Cowling, T.G., 1938, MNRAS, 98, 734

Giménez, A., \& Garcia-Pelayo, J.M., 1983, Ap\&SS, 92, 203

Hut, P., 1981, A\&A, 99, 126

Irwin, J.B., 1952, ApJ, 116, 211

Mazeh, T., 2008, EASP, 29, 1

Naoz, S., 2016, ARA\&A, 54, 441

Söderhjelm, S., 1984, A\&A, 141, 232

Sterne, T.E., 1939, MNRAS, 99, 451

Tauris, T.M., \& van den Heuvel, E.P.J., 2014, ApJ, 781, L13

Tokovinin, A., Thomas, S., Sterzik, M., \& Udry, S., 2006, A\&A, 450, 681

Weinberg, S., 1972, Gravitation and Cosmology: Principles and Applications of the General Theory of Relativity (Wiley-VCH)

Zahn, J.-P., 2008, EASP, 29, 67 